\title{
NANOSTRUCTURED COMPOSITES: EFFECTIVE MECHANICAL PROPERTY DETERMINATION OF NANOTUBE BUNDLES
}

\author{
E. Saether* \\ NASA Langley Research Center, Hampton, VA 23681 \\ R. B. Pipes ${ }^{\dagger}$ \\ University of Akron, Akron, OH 44325 \\ S.J.V. Frankland ${ }^{\dagger \dagger}$ \\ ICASE, NASA Langley Research Center, Hampton, VA 23681
}

\begin{abstract}
Carbon nanotubes naturally tend to form crystals in the form of hexagonally packed bundles or ropes that should exhibit a transversely isotropic constitutive behavior. Although the intratube axial stiffness is on the order of $1 \mathrm{TPa}$ due to a strong network of delocalized bonds, the intertube cohesive strength is orders of magnitude less controlled by weak, nonbonding van der Waals interactions. An accurate determination of the effective mechanical properties of nanotube bundles is important to assess potential structural applications such as reinforcement in future composite material systems. A direct method for calculating effective material constants is developed in the present study. The Lennard-Jones potential is used to model the nonbonding cohesive forces. A complete set of transverse moduli are obtained and compared with existing data.
\end{abstract}

\section{Introduction}

Future nanostructured composite materials are expected to incorporate carbon nanotube reinforcement either dispersed individually or as nanofilamentary bundles or ropes yielding unprecedented mechanical properties. A carbon nanotube is a cylindrical molecule composed of single or multiple walls of graphene sheets. These sheets are, in turn, composed of hexagonal units or graphene rings of carbon atoms that are bonded through highly stable $\mathrm{sp}^{2}$ hybridized orbitals. A typical carbon nanotube is schematically

depicted in Figure 1 while Figure 2 contains a crosssection of a bundle ensemble of individual nanotubes ${ }^{1}$.

Numerous studies have been made to analytically and experimentally determine the elastic properties of individual nanotubes. Axial Young's moduli on the order of $1 \mathrm{TPa}$ have been measured using atomic force microscopy $(\mathrm{AFM})^{2}$ and thermal vibrations ${ }^{3}$. Analytical studies have utilized ab initio calculations ${ }^{4}$, tightbinding methods, molecular dynamic simulations $(\mathrm{MD})^{6}$ and lattice dynamics ${ }^{7.8}$. These elastic properties are entirely based on the strong intratube valence forces of the carbon-carbon bonds.

Nanotube ensembles, however, typically form hexagonally packed crystal configurations in which the

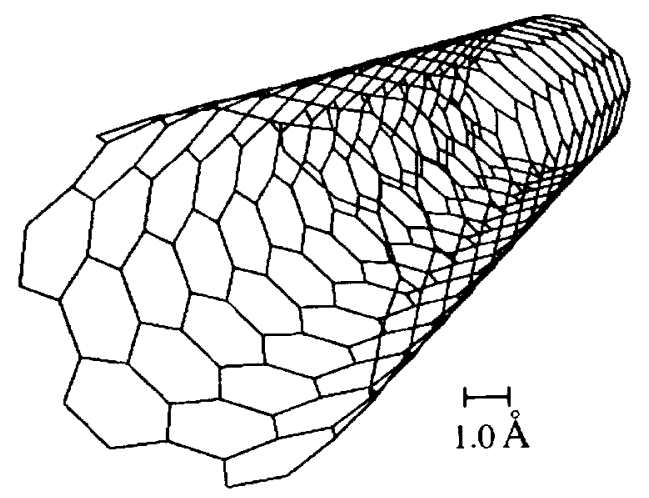

Figure 1. Single-walled nanotube.

\footnotetext{
* Aerospace Engineer, Analytical and Computational Methods Branch. Member, AIAA.

$t$ Goodyear Distinguished Professor, Polymer Engineering Academic Center.

${ }^{\dagger \dagger}$ Staff Scientist, ICASE.
}

Copyright $\odot 2002$ by the American Institute of Aeronautics and Astronautics, Inc. No copyright is asserted in the United States under Title 17, U.S. Code. The U.S. Government has a royalty-free license to exercise all rights under the copyright claimed herein for Government Purposes. All other rights are reserved by the copyright owner. 
intertube force interactions are due exclusively to nonbonding van der Waals effects which are much weaker than the valence forces and are highly nonlinear. Less consideration has been given to the transverse mechanical properties of nanotube bundles which depend on a good description of these non-bonding interactions. These intertube cohesive properties are of special interest for use in predicting the properties of carbon nanotube polymer composites ${ }^{9}$ and fibers of woven nanotubes ${ }^{10}$. Selected moduli of nanotube ropes have been calculated with a continuum model based on the integrated average of the discrete Lennard-Jones potential $^{11}$, MD simulation using the Tersoff-Brenner potential $^{12}$ and lattice dynamic methods ${ }^{13,14}$.

For the present study, a direct summation of atompair potentials is developed to avoid any simplifications made to the nonlinear van der Waals interactions and to permit optimum flexibility in representing discontinuous or curved nanotubes. Additionally, a direct method permits irregular and disrupted nanotube lattices to be modeled to simulate damage. Because the fundamental constituents of nanotube bundles are only resolvable at nanometer length scales, analyses to predict macroscopic properties must necessarily merge concepts and techniques from continuum elasticity theory and discrete molecular simulation. The methodology developed herein combines a unit cell continuum model with molecular static calculations to determine effective moduli in aligned carbon nanotube bundles.

The Lennard-Jones potential is utilized to simulate the van der Waals interaction forces among carbon atom-pairs in aligned carbon nanotube arrays. An achiral "zig-zag" configuration is assumed for the carbon nanotubes and the tube radius is assumed small such that the cross-section can be considered rigid.

The objective of this work is to formulate a molecular mechanics model to understand and predict the elastic properties of carbon nanotube bundles. This method utilizes a unit cell approach for determining selected transverse moduli of a hexagonal crystal of aligned nanotubes. The predicted moduli are shown to exhibit the transverse isotropy anticipated for a material possessing hexagonal symmetry. The predicted moduli are contrasted with available published data.

\section{Modified unit cell formulation}

In elasticity analyses, the method of unit cells has been developed to determine the effective properties of

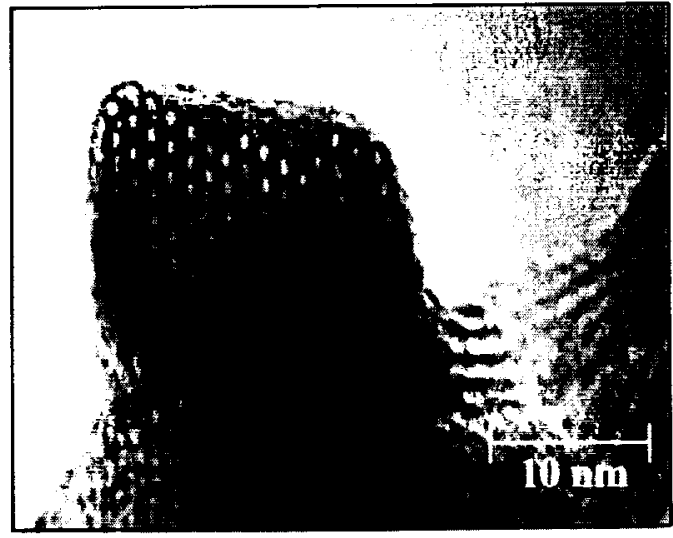

Figure 2. Typical nanotube bundle .

heterogeneous materials by identifying and analyzing convenient domains of repeating microstructure. Applied to the determination of effective continuum elastic moduli of bundles of aligned carbon nanotubes, a minimal repeating unit of nanotubes is defined and subjected to continuous field deformation modes. An assemblage of a primary unit cell of nanotubes with surrounding image regions that are required in applying periodic boundary conditions $(\mathrm{pbc}$ ) is presented in Figure 3. In this approach the nanotubes are regarded as sources of potential energy that require interactions with nanotubes outside the unit cell. Under periodic boundary conditions, cells of nanotubes in the transverse plane and nanotube segments in the axial dimension are treated as images of the constituents within the cell and used in the calculation of potential energy. This permits interactions between atom-pairs across the boundary to avoid introducing discontinuities in the force field. In general, these conditions ensure conservation of mass and energy, avoid surface or boundary effects, and mathematically give the primary unit cell a strict periodicity such that it can be considered to represent an infinite ensemble of molecules.

The basic approach of subjecting a molecular ensemble to fundamental strain modes and recovering effective moduli from energy measures has been used in molecular dynamic simulations ${ }^{15,16}$. By combining concepts from continuum elasticity and molecular dynamics, these representative units will be referred to herein as 'pbc-unit cells'. Pbc-unit cells can be constructed of arbitrary order but, with a proper definition of the repeat geometry, the unit energy of the primitive cell remains the same. Therefore the lowest order cell is used for computations. A minimum-order hexagonal pbc-unit cell is shown in Figure 4. 
The initial equilibrium configuration of the hexagonal unit cell is determined by minimizing the energy of the system as the nanotubes are moved radially outward from a fixed center. This establishes the equilibrium radius, $R_{e q p}$, and the nanotube center-tocenter separation distance, $S$, as shown in Figure 5.

A rigorous definition of the pbc-unit cell dimensions is required to ensure invariance of the unit energy with cell size. The required planar area of the pbc-unit cell is given by

$$
A_{\text {cell }}=\frac{3}{2} M \sqrt{3} R_{\text {eq }}^{2}
$$

where $M$ is the number of nanotubes within the unit cell. The effective depth of the pbc-unit cell is obtained by first selecting a number of repeat units (cicumferential rings of graphene), $K_{\text {seg }}$, and adding one additional unit to account for image segments in the axial dimension. The total area of the enclosed graphene units is then equated to the surface area of a perfect cylinder having the same radius as the nanotube. This is expressed as

$$
N A_{\text {gu }}\left(K_{s e q}+1\right)=2 \pi R_{n t} d_{e f f}
$$

where $N$ is the number of graphene units around the nanotube circumference, $A_{g u}$ is the area of an individual graphene unit, and $R_{n}$ is the nanotube radius. The radius of an $(\mathrm{N}, 0)$ nanotube can be derived as

$$
R_{n t}=\frac{\sqrt{3}}{4} b \operatorname{Sec}\left[\frac{\pi}{2}\left(1-\frac{1}{N}\right)\right]
$$

where $b$ is the carbon-carbon bond length. With the area of a graphene unit given by $A_{g u}=3 \sqrt{3} b^{2} / 2$, Equation (2) yields the effective depth of the pbc-unit cell as

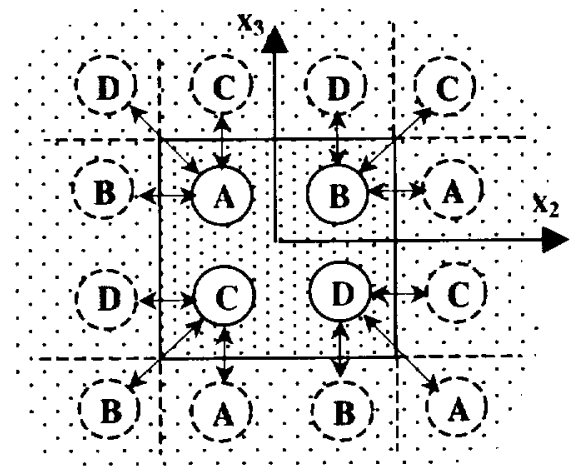

a) $(2,3)$-plane section

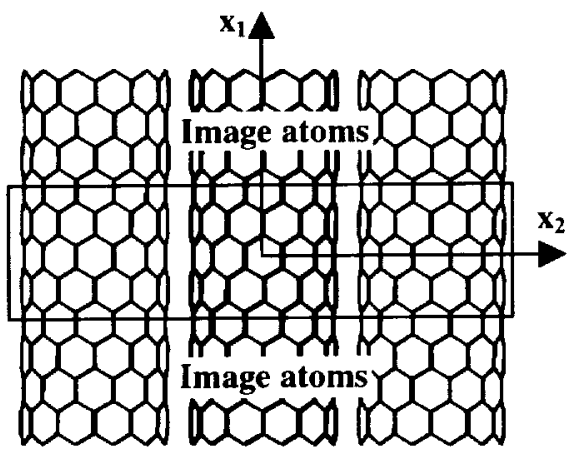

b) (1,2)-plane section

Figure 3. Pbc-unit cell showing outside periodic image nanotubes within the $(2,3)$-plane and image sections of nanotubes in the $(1,2)$-plane.

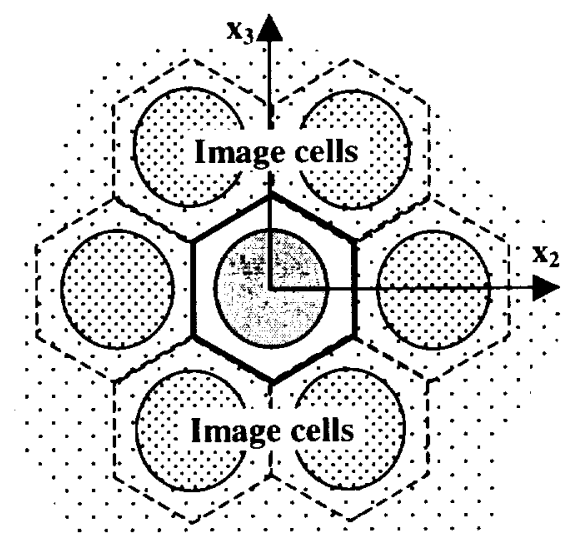

Figure 4. Miminal hexagonal pbc-unit cell with surrounding image cells.

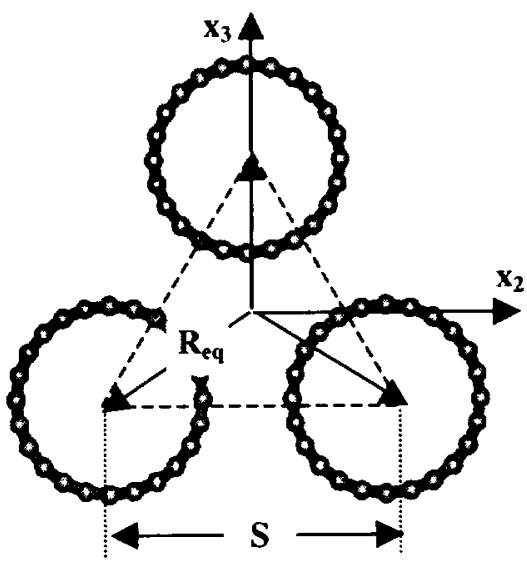

Figure 5. Equilibrium radius definition for a hexagonal cell. 


$$
\mathrm{d}_{\mathrm{eff}}=\frac{3}{\pi} \mathrm{Nb} \operatorname{Cos}\left[\frac{\pi}{2}\left(1-\frac{1}{\mathrm{~N}}\right)\right]\left(\mathrm{K}_{\mathrm{seF}}+1\right)
$$

\section{$\underline{\text { Material constitutive relationship }}$}

The force field within a nanotube crystal consists of a combination of strong linear bonding forces acting within the nanotube and weak non-bonding forces acting between adjacent nanotubes. This disparity between the magnitude of interatomic forces leads to a highly anisotropic constitutive relation. The hexagonal symmetry of the nanotube crystal dictates a transversely isotropic stress-strain relation given by

$$
\begin{gathered}
\sigma_{11}=C_{11} \varepsilon_{11}+C_{12} \varepsilon_{22}+C_{12} \varepsilon_{33} \\
\sigma_{22}=C_{12} \varepsilon_{11}+C_{22} \varepsilon_{22}+C_{23} \varepsilon_{33} \\
\sigma_{33}=C_{12} \varepsilon_{11}+C_{23} \varepsilon_{22}+C_{22} \varepsilon_{33} \\
\sigma_{12}=2 C_{44} \varepsilon_{12} \\
\sigma_{13}=2 C_{44} \varepsilon_{13} \\
\sigma_{23}=\left(C_{22}-C_{23}\right) \varepsilon_{23}
\end{gathered}
$$

Additional relationships between the material constants are given in Reference 17 for a hexagonal system as

$$
\begin{aligned}
& \mathrm{K}_{23}=\left(\mathrm{C}_{22}+\mathrm{C}_{23}\right) / 2 \\
& \mathrm{G}_{23}=\left(\mathrm{C}_{22}-\mathrm{C}_{23}\right) / 2
\end{aligned}
$$

This material is uniquely defined by five independent quantities where the ' $I$ ' direction is directed along the tube axis. The current effort will focus on the prediction of normal and shear properties in the transverse plane.

\section{Analysis methodology}

The methodology used to determine selected nanotube crystal properties involves defining an appropriate pbc-unit cell, applying selected strain modes to the crystal, and computing the potential energy due to atom-pair interactions as a function of the deformation kinematics. Effective elastic constants are then determined from the variation in the system potential energy as

$$
C_{13}=\left(\frac{1}{V}\right) \frac{\partial^{2} \Phi}{\partial \varepsilon_{1} \partial \varepsilon_{1}}
$$

where $C_{i j}$ is the material stiffness, $V$ is the pbc-unit cell volume, $\Phi$ is the potential energy, and $\varepsilon_{k}$ is an applied strain mode.

Strain modes are applied to the nanotubes in the crystal by the imposition of specific deformation fields. The $G_{23}$ shear modulus for a hexagonally packed nanotube array is calculated using a pbc-unit cell subjected to a pure shear strain mode as shown in Figure 6. The magnitude of the shear strain is given by twice the shear angle or $\gamma_{23}=2 \theta$. During a progressive deformation with increasing $\theta$, the potential energy is computed by summing all atom-pair interactions between adjacent nanotubes. The $\mathrm{G}_{23}$ shear modulus is then obtained from the elastic strain energy, $\Phi$, using a finite difference approximation as

$$
\mathrm{G}_{23}=\frac{1}{V_{\mathrm{ol}}} \frac{\partial^{2} \Phi}{\partial \gamma_{23}^{2}}=\frac{\Delta_{2} \Phi}{A_{\mathrm{exll}} \mathrm{d}_{\mathrm{etl}}\left(\Delta_{1} \gamma_{23}\right)^{2}}
$$

where $\mathrm{A}_{\text {cell }}$ is the planar area of the pbc-unit cell, $\mathrm{d}_{\mathrm{eff}}$ is the effective length of the nanotubes, $\Delta_{1}$ and $\Delta_{2}$ are first and second order central difference operators, respectively .

The bulk modulus is computed by applying a dilatational strain as shown in Figure 7 . Because the strain in the axial dimension, $\varepsilon_{11}$, is assumed to be zero, the dilation is defined as $\mathrm{e}=\varepsilon_{22}+\varepsilon_{33}$ with $\varepsilon_{22}=\varepsilon_{33}=\varepsilon$. The bulk modulus is obtained by

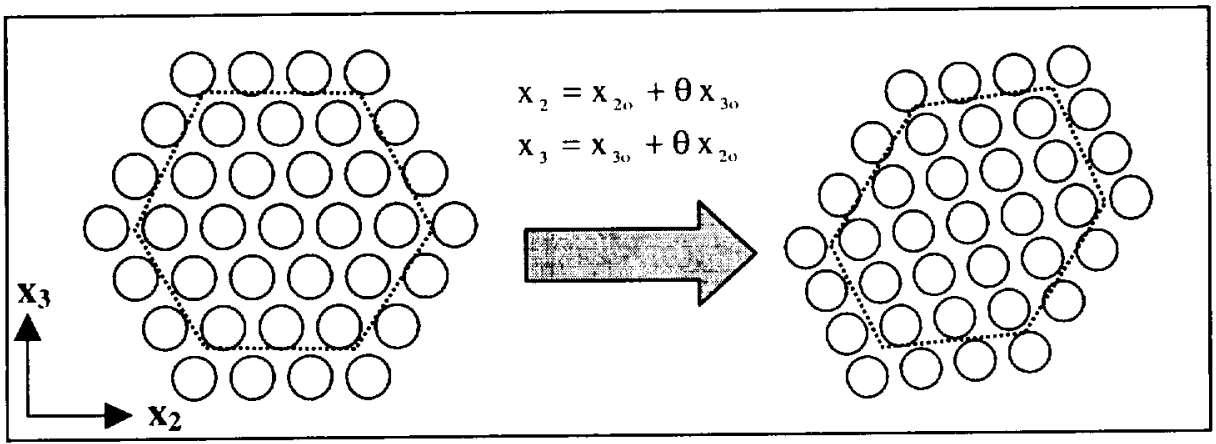

Figure 6. Imposed shear deformation on hexagonally packed nanotube array. 
applying Equation (7) using a strain given by $2 \varepsilon$. The calculation of the $E_{22}$ Young's modulus and the Poisson's ratio $v_{23}$ is performed by applying $\varepsilon_{22}$ strain increments in the 2-direction and repositioning of the tubes in the 3-direction to minimize the energy. The transverse repositioning of the tubes perpendicular to the load axis directly gives a measure of the Poisson contraction/expansion yielding $\varepsilon_{23}$. This is depicted in Figure 8. interactions. The Lennard-Jones or ' $6-12^{\prime}$ potential energy function is given by

$$
\Phi=4 \varsigma\left[\left(\frac{\alpha}{r_{11}}\right)^{12}-\left(\frac{\alpha}{r_{11}}\right)^{6}\right]
$$

where $\zeta$ is the depth of the energy well, $\alpha$ is the van

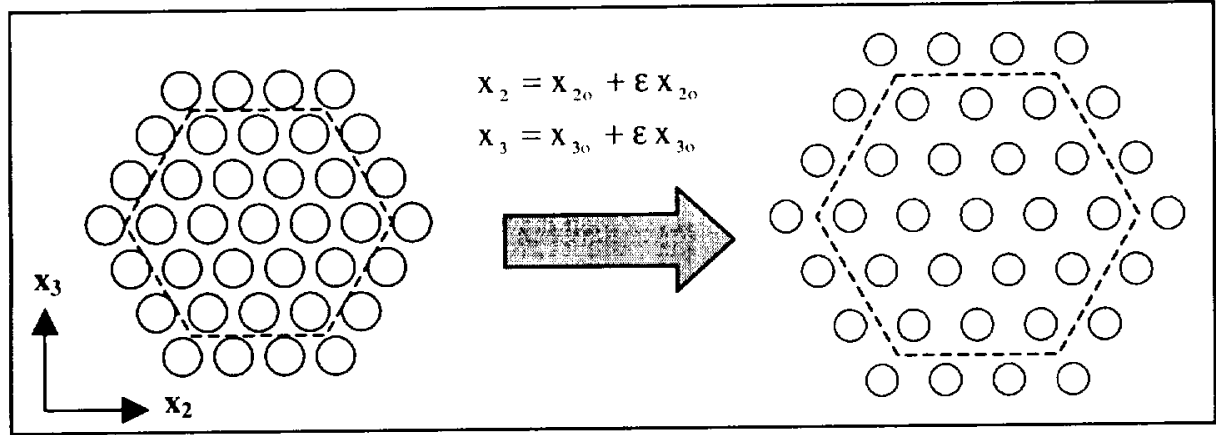

Figure 7. Imposed dilatational strain on hexagonally packed nanotube array.

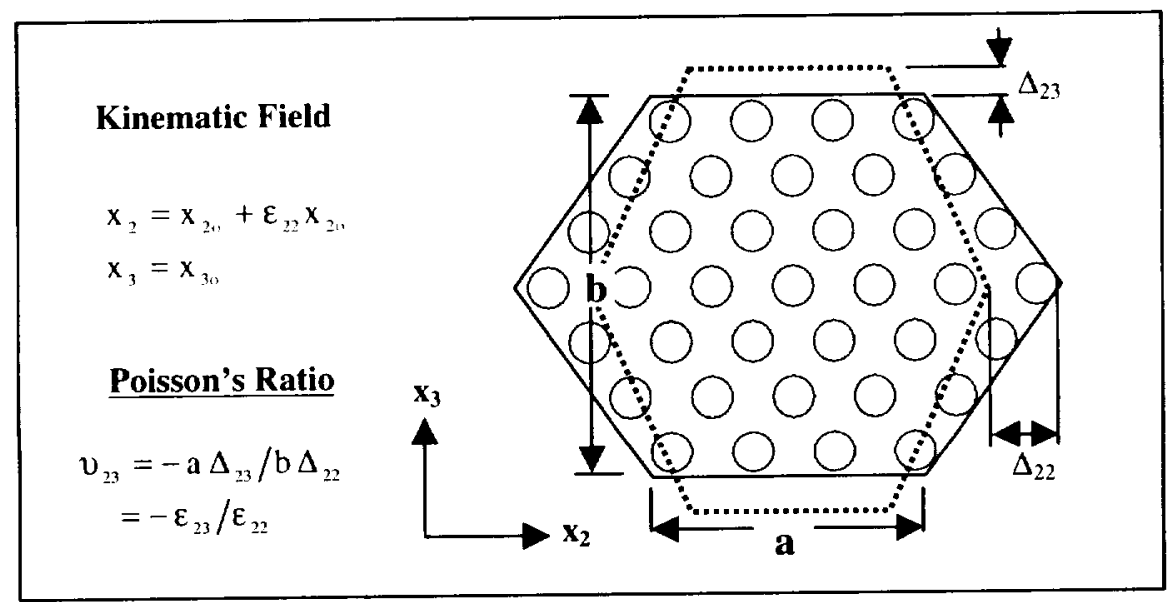

Figure 8. Calculation of Poisson's ratio.

The $\mathrm{E}_{22}$ modulus is obtained using Equation (7) and the Poisson ratio is determined as $v_{23}=-\varepsilon_{23} / \varepsilon_{22}$. The $\mathrm{C}_{22}$ stiffness coefficient is obtained by applying the same deformation mode defined in Figure 8 , but no energy minimization is performed to induce a transverse Poisson deformation.

\section{Potential energy calculations}

The intertube forces are typically modeled by the Lennard-Jones potential to represent van der Waals der Waals radius, and $r_{i j}$ is the separation distance between the $i^{\text {th }}$ and $j^{\text {th }}$ atoms in a pair. The $r_{i j}^{-6}$ term represents the attractive contribution to the van der Waals forces between neutral molecules. It includes permanent dipole-dipole interactions, the induction effect of permanent dipoles, and instantaneous dipole induced dipole interactions which are sometimes referred to as the London dispersion forces. The other component of the van der Waals interactions mimics the repulsion between overlapping electron clouds and is modeled by the $r_{1 j}{ }^{-12}$ term which is short ranged ${ }^{18,19}$. 


\section{$\underline{\text { Results and discussion }}$}

In the present study, a crystal of single-walled nanotubes is analyzed. Each nanotube has a diameter of $0.94 \mathrm{~nm}$ and an achiral zig-zag conformation with 12 graphene units around the circumference. The carboncarbon bond length is prescribed as $1.42 \AA$. Nanotubes of this size may be considered rigid in the transverse direction ${ }^{12.13}$. Therefore, the only degree of freedom included in the deformation kinematics is the relative motion of the nanotube center, and the only contribution to the potential energy changes with imposed motion is computed using the Lennard-Jones potential. The parameters used in the Lennard-Jones potential are $\varsigma=34.0(\mathrm{~K})$ and $\alpha=0.3406 \mathrm{~nm}^{13,20}$. A comparison between predicted elastic moduli using the current direct method and results obtained using alternate approaches is presented in Table 1. From the limited published results it is clear that there is a wide variation in predicted elastic moduli for nanotube bundles. $v_{23}$. Substituting the calculated values for the Young's modulus $E_{22}$ and Poisson's ratio $v_{23}$ into the resulting expression, the transverse normal stiffness is obtained as

$$
\mathrm{C}_{22}=\frac{\mathrm{E}_{22}}{1-\mathrm{v}_{23}^{2}}=\frac{60.3}{1-0.34^{2}}=68.2 \mathrm{GPa}
$$

which closely agrees with the independently calculated value of $\mathrm{C}_{22}$. Next, computing $\mathrm{C}_{23}$ as $\mathrm{v}_{23} \mathrm{C}_{22}=23.2$ GPa and applying the relations given by Equations (6), it is found that the transverse plane stiffnesses are recoverable from the computed shear and bulk moduli as

$$
\begin{aligned}
& \mathrm{C}_{22}=\mathrm{K}_{23}+\mathrm{G}_{23}=45.8+22.5=68.3 \mathrm{GPa} \\
& \mathrm{C}_{23}=\mathrm{K}_{23}-\mathrm{G}_{23}=45.8-22.5=23.3 \mathrm{GPa}
\end{aligned}
$$

Thus, the computed elastic constants are completely self-consistent for a transversely isotropic material as required. The values reported in Reference 13 (Table 1)

Table 1. Comparison of predicted elastic constants.

\begin{tabular}{|l|c|c|c|c|}
\hline \multicolumn{1}{|c|}{ Elastic Constant } & Direct Method & Reference $13^{*}$ & Reference $14^{* *}$ & Reference 12 \\
\hline Bulk Modulus $\mathrm{K}_{23}(\mathrm{GPa})$ & 45.8 & 42.0 & 18.0 & 33.6 \\
\hline Shear Modulus $\mathrm{G}_{23}(\mathrm{GPa})$ & 22.5 & $5.3^{*}$ & -- & -- \\
\hline Young's Modulus $\mathrm{E}_{22}(\mathrm{GPa})$ & 60.3 & 17.0 & -- & -- \\
\hline Normal Stiffness $\mathrm{C}_{22}(\mathrm{GPa})$ & 68.3 & 42.0 & 78.0 & -- \\
\hline Poisson Ratio $v_{23}$ & 0.34 & 0.75 & -- & -- \\
\hline
\end{tabular}

*Value derived using relationship in Equation (6).

${ }^{* *}$ Results generated using $(7,7)$ helical nanotubes with diameter $=0.94 \mathrm{~nm}$.

All the results listed in Table 1 for the present analysis were computed independently, none were derived from a subset of other values. For a 3-D solid exhibiting hexagonal symmetry, the expression for the transverse Young's modulus, $E_{22}$, is given by ${ }^{13}$

$$
E_{22}=\frac{\left(C_{33}-C_{32}\right)\left[\left(C_{33}+C_{32}\right) C_{11}-2 C_{31}^{2}\right]}{\left(C_{33} C_{11}-C_{13}^{2}\right)}
$$

Because the axial modulus of the nanotubes in the crystal is generally two orders of magnitude greater than the transverse moduli, we may take $C_{11} \gg C_{33}, C_{32}, C_{31}$. Using the relationship $C_{23}=v_{23} C_{22}$ and equating Poisson ratios under transverse plane isotropy as $v_{23}=v_{32}$, Equation (10) reduces to a simple relationship between $\mathrm{C}_{22}, \mathrm{E}_{22}$ and are also self-consistent for transverse isotropy and compare favorably with the present analysis for the prediction of the bulk modulus, $\mathrm{K}_{23}$. In contrast, an analytical result presented in Reference 12 (Table 1) is slightly lower. The calculation of the normal stiffness, $\mathrm{C}_{22}$, which is obtained from applying a similar deformation as that used to compute the bulk modulus (applying only $\varepsilon_{22}$ instead of both $\varepsilon_{22}$ and $\varepsilon_{33}$ with $\varepsilon_{22}=$ $\varepsilon_{33}$ ), is distinctly different from Reference 13 . The present analysis yields a stiffness closer to that of Reference 14 (Table 1) in which nanotubes of the same radius were used but with different helicity. The effect of helicity is discussed in Reference 13 in which it is shown for several cases that the configuration of graphene units on the nanotube surface has a negligible effect for small radius tubes ( $\mathrm{R}<16 \AA$ ). Furthermore, from results presented in Reference 13, $C_{23}$ may be 
calculated as $\mathrm{C}_{23}=\mathrm{v}_{23} \mathrm{C}_{22}=31.5 \mathrm{GPa}$. If one applies the first of the relations given in Equations (6), one obtains a value for $\mathrm{G}_{23}$ of $5.3 \mathrm{GPa}$. This shear modulus is comparable to the shear modulus associated with parallel planes in graphite which is experimentally measured as $4.0 \mathrm{GPa}^{21,22}$ and indicates a significant discrepancy between predictions using the present method and the lattice dynamics approach used in Reference 13. In the direct summation and lattice dynamic methods, the physics of cohesion are identically represented by the same parameterization of the Lennard-Jones potential. Possible differences in predictions using lattice dynamics may be due to the inherent integral averaging of force constants used in the lattice dynamical matrix and the a priori selection of interacting nearest-neighbor atoms used in defining the primitive lattice cell, both of which are avoided in the direct method.

A potential source of inaccuracy effecting all methods is the form of the Lennard-Jones potential function itself. The Lennard-Jones potential was originally developed for noble gases and is known to produce poor results in other applications including graphite. It gives good results for the $C_{33}$ modulus (interplanar separation), but the $\mathrm{C}_{44}$ parallel plane shear modulus is underpredicted by an order of magnitude ${ }^{22}$. Alternative potentials have been proposed ${ }^{23}$ that account for the delocalized electronic configuration of graphite and yield accurate predictions for both the transverse normal and shear moduli. Additional study is warranted to assess the spatial interactions of delocalized bonds in carbon nanotubes that may be underestimated using a spherical Lennard-Jones model.

\section{Conclusions}

A consistent method has been formulated for and applied to computing effective transverse mechanical properties of nanotube crystals. The method is based on specifying a unit cell configuration with periodic boundary conditions, applying a deformation field associated with a particular strain mode, and utilizing a direct summation procedure to compute changes in potential energy from which an effective elastic modulus may be obtained. The disparity between reported predictions of mechanical properties that depend exclusively on van der Waals cohesion and the paucity of available experimental data suggest that much additional investigation is warranted in this area. The development of a more realistic representation of van der Waals interactions between nanotube surfaces may be required to correlate analytical predictions with future experimental measurements of nanotube crystal properties.

\section{Acknowledgments}

The authors would like to thank Prof. V. Popov at the University of Sofia for helpful discussions during the course of this work. This research was partially supported by the National Aeronautics and Space Administration under NASA Contract No. NAS197046 while S. J. V. Frankland was in residence at ICASE, NASA Langley Research Center, Hampton, VA 23681-2199.

\section{$\underline{\text { References }}$}

1. Thess, A., Lee R., Nikolaev, P., Dai, H., Petit, P., Robert, J., Xu, C., Lee, Y. H., Kim, S. G., Rinzler, A. G., Colbert, D. T., Scuseria, G. E., Tomanek, D., Fischer, J. E. and Smalley, R. E. "Crystalline Ropes of Metalic Carbon Nanotubes," Science Vol. 273, 1996, pp. 483-487.

2. Salvetat, J.P., Briggs, G.A.D., Bonard, J.M, Bacsa, R.R., Kulik, A.J., Stockli, T., Burnham, N.A. and Forro, L., "Elastic and Shear Moduli of SingleWalled Carbon Nanotubes," Phys. Rev. Lett, Vol. 82, 1999, pp. 944-947.

3. Krishnan, A., Dujardin, E., Ebbesen, T.W., Yianilos, P.N. and Treacy, M.M.J., "Young's Modulus of Single-Walled Nanotubes," Phys. Rev. $B$, Vol. 58, 1998, pp. 14013-14019

4. Sanchez-Portal, D., Artacho, E., Soler, J.M., Rubio. A. and Ordejon, P., "Ab Initio Structural, Elastic, and Virbrational Properties of Carbon Nanotubes." Phys. Rev. B, Vol. 59, 1999, pp.12678-12688.

5. Hernandez, E., Goze, C., Bernier, P. and Rubio, A., "Elastic Properties of $\mathrm{C}$ and $\mathrm{B}_{\mathrm{x}} \mathrm{C}_{\mathrm{y}} \mathrm{N}_{\mathrm{z}}$ Composite Nanotubes," Phys. Rev. Lett., Vol. 80, 1998, pp. 4502-4505.

6. Cornwell, C.F. and Wille, L.T., "Elastic Properties of Single-Walled Carbon Nanotubes in Compression," Solid State Commun., Vol. 101, 1997, pp. 555-558.

7. Popov, V.N., Van Doren, V.E. and Balkanski, M., "Elastic Properties of Single-Walled Carbon Nanotubes," Phys. Rev. B, Vol. 61, 2000, pp. 30783084.

8. Lu, J.P., "Elastic Properties of Single and Multilayered Nanotubes," J. Phys. Chem. Solids, Vol. 58, 1997, pp. 1649-1652.

9. Frankland, S.J.V., Caglar, A., Brenner, D.W. and Griebel, M., "Molecular Simulation of the Influence of Chemical Cross-Links on the Shear Strength of Carbon Nanotube-Polymer Interfaces," Submitted to J. Phys. Chem. B, 2002. 
10. Pipes, R.B. and Hubert, P., "Helical Carbon Nanotube Arrays: mechanical Properties," Comp. Science and Tech., (in press), 2002.

11. Girifalco, L.A., Hodak, M. and Lee, R.S., "Carbon Nanotubes, Buckyballs, Ropes, and a Universal Graphitic Potential," Phys. Rev. B, Vol. 62, 2000, pp. 13104-13110.

12. Tersoff, J. and Ruoff, R.S., "Structural Properties of a Carbon-Nanotube Crystal," Phys. Rev. Lett., Vol. 73, 1994, pp. 676-679.

13. Popov, V.N., Van Doren, V.E. and Balkanski, M., "Elastic Properties of Crystals of Single-Walled Carbon Nanotubes," Solid State Commun., Vol. 114, 2000, pp. 395-399.

14. Lu, J.P., "Elastic Properties of Carbon Nanotubes and Nanoropes," Phys. Rev. B, Vol. 79, 1997, pp. 1297-1300.

15. Theodorou, D.N. and Suter, U.W., "Atomistic Modeling of Mechanical Properties of Polymeric Glasses," Macromolecules, Vol. 19, 1986, pp. 139154.

16. Fan, C.F. and Hsu, S.L., "Application of the Molecular Simulation Technique to Characterize the Structure and Properties of an Aromatic Polysulfone System. 2 Mechanical and Thermal Properties," Macromolecules, Vol. 25, 1992, pp. 265-270.

17. Hashin, Z. and Rosen, B.W., "The Elastic Moduli of Fiber-Reinforced Materials," J. Appl.. Mech., 1964, pp. 223-232.

18. Allen, M.P., and Tildesley, D.J., Computer Simulation of Liquids, Claredon Press, 1987.

19. Moore, W. J., Physical Chemistry, $4^{\text {th }}$ ed. Prentice Hall Englewood Cliffs, New Jersey, 1972, pp. 913915.

20. Lu, J.P. and Yang, W., "The Shape of Large Single- and Multiple-Shell Fullerenes," Phys. Rev. B., Vol. 49, 1994, pp. 11421-11424.

21. Kelly, B.T. and Duff, M.J., "On the Validity of Lennard-Jones Potentials for the Calculation of Elastic Properties of a Graphite Crystal," Carbon, Vol. 8, 1970, pp. 77-85.

22. Green, J.F., Bolland, T.K. and Bolland, J.W., "Lennard-Jones Interactions for Hexagonal Layered Crystals," J. Chem. Phys., Vol. 61, 1974, pp. 1637-1646.

23. Kolmogorov, A.N. and Crespi, V.H., "Smoothest Bearings: Interlayer Sliding in Multiwalled Carbon Nanotubes," Phys. Rev. Lett., Vol. 85, 2000, pp. 4727-4730. 